\title{
ALMOST COMPLETELY DECOMPOSABLE TORSION FREE ABELIAN GROUPS
}

\author{
E. L. LADY
}

ABSTRACT. A finite rank torsion free abelian group $G$ is almost completely decomposable if there exists a completely decomposable subgroup $C$ with finite index in $G$. The minimum of $[G: C]$ over all completely decomposable subgroups $C$ of $G$ is denoted by $i(G)$. An almost completely decomposable group $G$ has, up to isomorphism, only finitely many summands. If $i(G)$ is a prime power, then the rank 1 summands in any decomposition of $G$ as a direct sum of indecomposable groups are uniquely determined. If $G$ and $H$ are almost completely decomposable groups, then the following statements are equivalent: (i) $G \oplus L \approx H \oplus L$ for some finite rank torsion free abelian group $L$. (ii) $i(G)=i(H)$ and $H$ contains a subgroup $G^{\prime}$ isomorphic to $G$ such that $\left[H: G^{\prime}\right]$ is finite and prime to $i(G)$. (iii) $G \oplus L \approx H \oplus L$ where $L$ is isomorphic to a completely decomposable subgroup with finite index in $G$.

A finite rank torsion free abelian group $G$ is almost completely decomposable if there is a completely decomposable subgroup $C$ having finite index in $G$. It is well known that direct sum decompositions of such groups need not be unique. In fact, this class of groups is the source of all the most familiar examples of nonunique decompositions of finite rank torsion free abelian groups. This paper will show, however, that the situation is not completely unruly. We show that in certain situations cancellation of direct summands is possible. We show that a maximal completely decomposable summand is unique up to isomorphism. We show that an almost completely decomposable group $G$ has, up to isomorphism, only finite many summands. We show that there are only finitely many groups $H$ for which there exists a finite rank torsion free abelian group $L$ such that $G \oplus L \approx H \oplus L$. Theorem 11 characterizes such groups $H$.

All groups in this paper, unless indicated otherwise, are finite rank torsion free abelian groups. In general, we will follow the notation and conven-

Presented to the Society, February 2, 1973; received by the editors June 18, 1973. AMS (MOS) subject classifications (1970). Primary $20 \mathrm{~K} 15$.

Key words and phrases. Almost completely decomposable group, cancellation property, regulating subgroup, $K$-equivalence. 
tions of [2]. In particular, if $H$ is a subgroup of $G, H_{*}$ denotes the smallest pure subgroup of $G$ containing $H$. An isomorphism class of rank 1 groups is called a type. The type determined by a rank 1 group $A$ is denoted by $\mathfrak{t}(A)$. The set of types is partially ordered by writing $\mathfrak{t}(A) \leq \mathfrak{t}(B)$ if $\operatorname{Hom}(A, B) \neq$ 0 . If $x \in G$, then $\mathfrak{t}(x)$ denotes $\mathfrak{t}\left(\langle x\rangle_{*}\right)$. We say that $G$ is homogeneous of type $\tau$ if $\mathfrak{t}(x)=\tau$ for all $0 \neq x \in G$, and that $G$ is completely decomposable if $G$ is a direct sum of rank 1 groups. If $G$ is completely decomposable, then $G_{\tau}$ will denote a maximal $\tau$-homogeneous summand of $G$, so that $G=\bigoplus \Sigma_{\tau} G_{\tau}$.

If $\tau$ is a type, then $G(\tau)$ is the subgroup of $G$ consisting of those $x$ with $\mathfrak{t}(x) \geq \tau$. We will use $G^{\#}(\tau)$ to denote the pure subgroup of $G$ generated by all $G(\sigma)$ for $\sigma>r$, and $G(S)$ to denote the subgroup generated by all $G(\sigma)$ with $\sigma \in S$, where $S$ is a set of types.

Torsion free groups $G$ and $H$ are quasi-isomorphic if $G$ is isomorphic to a subgroup of finite index in $H$. This is equivalent to the existence of monomorphisms from $G$ into $H$ and from $H$ into $G$. We will call $G$ and $H$ $K$-equivalent if $G \oplus L \approx H \oplus L$ for some finite rank torsion free abelian group $L$.

1. Regulating subgroups. The concept of a regulating subgroup of an almost completely decomposable group is the key to everything that follows.

Definition. If $G$ is an almost completely decomposable group, $i(G)$ will denote the minimum of $[G: C]$ where $C$ ranges over the set of all completely decomposable subgroups of $G$. A regulating subgroup of $G$ is a completely decomposable subgroup $C$ with finite index in $G$ such that, for each type $\tau$, $G(\tau)=C_{\tau} \oplus G^{\sharp}(\tau)$.

Notice that if $C$ is a regulating subgroup of $G$, then, for each type $\tau$, $C_{\tau}$ is pure in $G$. The existence of regulating subgroups follows from the following theorem:

Theorem 1. A completely decomposable subgroup $C$ of an almost completely decomposable group $G$ is a regulating subgroup if and only if $[G: C]$ $=i(G)$. If $D$ is any completely decomposable subgroup of $G$, then $[G: D]$ is a multiple of $i(G)$.

Proof. We show first that if $C$ and $D$ are regulating subgroups of $G$, then $[G: C]=[G: D]$. Notice that $C$ and $D$ are quasi-isomorphic, hence isomorphic. Choose a minimal type $\tau$ such that $C_{\tau} \neq 0$ and let $S=\left\{\sigma \mid C_{\sigma} \neq 0, \sigma \neq \tau\right\}$, $C_{S}=\bigoplus \Sigma_{S} C_{\sigma}, D_{S}=\bigoplus \Sigma_{S} D_{\sigma}$. Then $C_{S}$ and $D_{S}$ are regulating subgroups of $G(S)_{*}$ so that, by induction, we may assume that $\left[G(S)_{*}: C_{S}\right]=\left[G(S)_{*}: D_{S}\right]$. Since $C_{\tau} \oplus G(S)_{*}=G(\tau)+G(S)_{*}$, we have 


$$
\begin{aligned}
{[G: C] } & =\left[G: C_{\tau} \oplus G(S)_{*}\right]\left[C_{\tau} \oplus G(S)_{*}: C\right] \\
& =\left[G: G(\tau)+G(S)_{*}\right]\left[G(S)_{*}: C_{S}\right]=\left[G: D_{\tau} \oplus G(S)_{*}\right]\left[G(S)_{*}: D_{S}\right]=[G: D] .
\end{aligned}
$$

It now suffices to show that if $D$ is a completely decomposable subgroup of $G$ and $D$ is not a regulating subgroup, then there exists a completely decomposable subgroup $C$ of $G$ so that $[G: D]$ is a proper multiple of $[G: C]$ (hence $[G: D] \neq i(G)$ ). Let $\tau$ and $S$ be chosen as before. The computation above shows that $[G: D]=\left[G: D_{\tau} \oplus G(S)_{*}\right]\left[G(S)_{*}: D_{S}\right]$. Choose, by induction, a regulating subgroup $C_{S}$ of $G(S)_{*}$ and choose by [2, Proposition 86.5, p. 114], a subgroup $C_{\tau}$ of $G$ so that $G(\tau)=C_{\tau} \oplus G^{\sharp}(\tau)$. Since $D$ is not a regulating subgroup, either $G(\tau) \neq D_{\tau} \oplus G^{\#}(\tau)$ and $\left[G: D_{\tau} \oplus G(S)_{*}\right]$ is a proper multiple of $\left[G: C_{\tau} \oplus G(S)_{*}\right]$ or $D_{S}$ is not a regulating subgroup of $G(S)_{*}$ and, by induction, $\left[G(S)_{*}: D_{S}\right]$ is a proper multiple of $\left[G(S)_{*}: C_{S}\right.$. In either case, $C_{\tau} \oplus C_{S}$ is the desired subgroup $C$.

Corollary 2. If $G$ and $H$ are $K$-equivalent almost completely decomposable groups, then $i(G)=i(H)$.

Proof. Let $C$ and $D$ be regulating subgroups of $G$ and $H$, and let $\tau$ and $S$ be as in the proof of Theorem 1. If $G \oplus L \approx H \oplus L$, it follows that $G /\left(G(\tau)+G(S)_{*}\right) \approx H /\left(H(\tau)+H(S)_{*}\right)$. Also $G(S)_{*} \oplus L(S)_{*} \approx H(S)_{*} \oplus L(S)_{*}$, hence $G(S)_{*}$ and $H(S)_{*}$ are $K$-equivalent, so by induction $\left[G(S)_{*}: C_{S}\right]=$ $\left[H(S)_{*}: D_{S}\right]$. Thus

$[G: C]=\left[G: G(\tau)+G(S)_{*}\right]\left[G(S)_{*}: C_{S}\right]=\left[H: H(\tau)+H(S)_{*}\right]\left[H(S)_{*}: D_{S}\right]=[H: D]$.

Remark. If $C$ and $D$ are regulating subgroups of $G$, it need not be the case that $G / C \approx G / D$. For instance, let $A, B$ and $H$ be noncomparable rank 1 groups and let $K$ be a rank 1 group with $\mathfrak{t}(K) \leq \inf \{\mathfrak{t}(A), \mathfrak{t}(B)\}$ and $\mathfrak{t}(K) \notin \mathfrak{t}(H)$. Let $p$ be a prime such that none of $A, B, H, K$ are $p$-divisible and choose $a, b, h, k$ in $A, B, H, K$ all with $p$-height 0 . Let $G$ be generated by $A, B, H, K$ and $(a+b) / p,(b+k) / p$. Let $K^{\prime}$ be the pure subgroup of $G$ generated by $k+(a+b) / p$. Then $C=A \oplus B \oplus H \oplus K$ and $D=A \oplus$ $B \oplus H \oplus K^{\prime}$ are regulating subgroups of $G$. But $G / C \approx Z(p) \oplus Z(p)$ whereas $G / D \approx Z\left(p^{2}\right)$.

Corollary 3. If $G$ and $H$ are almost completely decomposable groups, then $i(G \oplus H)=i(G) i(H)$.

Proof. If $C$ and $D$ are regulating subgroups of $G$ and $H$, then $C \oplus D$ is a regulating subgroup of $G \oplus H$, and $[G \oplus H: C \oplus D]=[G: C][H: D]$.

Corollary 4. An almost completely decomposable group $G$ has, up to 
isomorphism, only finitely many summands. There are only finitely many groups $H$ which are $K$-equivalent to $G$.

Proof. Let $n$ be a positive integer and $D$ a completely decomposable group. Then there are, up to isomorphism, only finitely many almost completely decomposable groups $H$ such that $i(H)=n$ and $D$ is a regulating subgroup of $H$. In fact, $H \approx n H$ and $n D \subseteq n H \subseteq D$. But $D / n D$ is a finite group, so there are only finitely many subgroups of $D$ containing $n D$. Now if $H$ is $K$-equivalent to $G$, then $i(G)=i(H)$ and $D$ is isomorphic to a regulating subgroup of $G$ (because $G$ and $H$ are quasi-isomorphic). On the other hand, if $H$ is a summand of $G$, then $i(H)$ is a divisor of $i(G)$ and $D$ is isomorphic to a summand of a regulating subgroup of $H$. So in either case, there are only finitely many such $H$.

2. Completely decomposable summands. In general, completely decomposable groups do not have the cancellation property. The following theorem shows, however, that cancellation is sometimes possible. This theorem is a special case of a result in [1].

Theorem 5. Let $G=A \oplus H=B \oplus C$ where $A$ is a $r$-homogeneous completely decomposable group and $H$ has no quasi-summands of type $\tau$. Then $B$ and $C$ have summands $B_{1}$ and $C_{1}$ so that $G=B_{1} \oplus C_{1} \oplus H$. In particular, if also $G \approx A \oplus H_{1}$, then $H \approx H_{1}$.

Outline of proof. Let $\alpha$ and $\beta$ be the projections onto $A$ and $B$. The hypothesis on $H$ implies that the restriction to $H(\tau)$ of $\alpha \beta$ must be zero Hence $H(\tau)$ is invariant under $\beta$ so that there exist summands $B_{1}$ and $C_{1}$ of $B(\tau)$ and $C(\tau)$ such that $G(\tau)=B_{1} \oplus C_{1} \oplus H(\tau)$. It follows that $G=B_{1} \oplus C_{1} \oplus H$.

It is well known that this theorem fails if we merely suppose that $H$ has no rank 1 summands of type 7 . (For instance, cf. [2, Theorem 90.4, p. 138].) For almost completely decomposable groups, however, we do have the following weaker theorem :

Theorem 6. If $G$ is an almost completely decomposable group and $G=$ $A \oplus H=B \oplus K$ where $A, B$ are $\tau$-homogeneous and $H, K$ have no rank 1 summands of type $\tau$, then $A \approx B$.

Proof. Note that $A$ and $B$ are necessarily completely decomposable groups. By $[2$, Lemma 86.8 , p. 115] $H \cap B$ is a $r$-homomoneous completely decomposable summand of $B$ and hence of $H$ (because $B$ is a summand of $G)$. Thus $H \cap B=0$ by the hypothesis on $H$. Thus the projection onto $A$ maps 
$B$ monomorphically into $A$, so rank $B \leq \operatorname{rank} A$. By symmetry, rank $A=$ rank $B$, so $A \approx B$.

Corollary 7. If $G$ is an almost completely decomposable group, then a maximal completely decomposable summand of $G$ is unique up to isomorphism.

It does not follow from Theorem 6 that any two decompositions of $G$ into a direct sum of indecomposable groups will involve the same number of summands of a given type $\tau$. This is because, as is well known, $G_{1} \oplus G_{2}$ may have a rank 1 summand even when neither $G_{1}$ nor $G_{2}$ do. However, this cannot occur if $i(G)$ is a prime power. The proof of this depends on the following technical lemma:

Lemma 8. Let $G$ be an almost completely decomposable group and $C$ a regulating subgroup, and suppose $i(G)=p^{k}$ for some prime $p$. For some type $\tau$, let $C_{1}$ be a rank 1 summand of $C_{\tau}$ and let $S=\{\sigma \mid \sigma \underline{\leftarrow} \tau\}$. Then $C_{1}$ is a summand of $G$ if and only if $C_{1} \oplus G(S)_{*}$ is a pure subgroup of G. Otherwise $\left(C_{1} \oplus G(S)\right)_{*} /\left(C_{1} \oplus G(S)_{*}\right)$ is a cyclic p-group.

Proof. Let $\phi:\left(C_{1} \oplus G(S)\right)_{*} \rightarrow C_{1}$ be defined by multiplication by $p^{k}$ followed by the projection of $C_{1} \oplus G(S)_{*}$ onto $C_{1}$. Since $C_{1}$ and $G(S)_{*}$ are pure, $\phi$ induces a monomorphism of $\left(C_{1} \oplus G(S)\right)_{*} /\left(C_{1} \oplus G(S)_{*}\right)$ into $C_{1} / p^{k} C_{1}$, which is a cyclic p-group. Now if $G=C_{1} \oplus H$, then $G(S)_{*} \subseteq H$, so $C_{1} \oplus G(S)_{*}$ is pure. Conversely, suppose that $C_{1} \oplus G(S)_{*}$ is pure. We may suppose that $C=C_{1} \oplus C_{2} \oplus C_{3}$ where $C_{2}$ has rank 1 and $\mathfrak{t}\left(C_{2}\right) \leq \tau$ and $C(S) \subseteq C_{3}$, since otherwise $G=C_{1} \oplus G(S)_{*}$. Then by induction on the rank of $G, C_{1}$ is a summand of $\left(C_{1} \oplus C_{3}\right)_{*}$ and $C_{3}$ may be chosen so that $\left(C_{1} \oplus C_{3}\right)_{*}=C_{1} \oplus C_{3 *}$. Then since $C_{2}$ and $C_{1} \oplus C_{3 *}^{3}$ are pure and $C_{2}$ has rank 1, it follows that $G /\left(C_{1} \oplus C_{2} \oplus C_{3 *}\right)$ is trivial or a cyclic $p$-group. In the former case we are done. Otherwise, let $g \in G$ be a representative for a generator of this group. Then, for some $s \leq k, p^{s} g=c_{1}+c_{2}+c_{3} \in C_{1} \oplus C_{2} \oplus C_{3 *}$ where both $c_{1}+$ $c_{3}$ and $c_{2}$ have $p$-height 0 and $c_{1} \neq 0$. Now since $t\left(C_{2}\right) \leq t\left(C_{1}\right)$, we have that $h t_{q}\left(c_{2}\right) \leq b t_{q}\left(c_{1}\right)$ for $q=p$, whenever $h t_{q}\left(c_{2}\right)=\infty$, and with finitely many exceptions otherwise. Choose $m$ so that $1+m p^{s}$ is divisible by large powers of the exceptional primes. Then $C_{1} \oplus C_{2} \subseteq C_{1} \oplus C_{2}^{\prime}$ where $C_{2}^{\prime}=$ $\left\langle c_{2}+\left(1+m p^{s}\right) c_{1}\right\rangle *$ Then $g+m c_{1} \in\left(C_{2}^{\prime} \oplus C_{3}\right)_{*}$ and $g+m c_{1}$ represents a generator of $G /\left(C_{1} \oplus C_{2} \oplus C_{3 *}\right)$. Hence $G=C_{1} \oplus\left(C_{2}^{\prime} \oplus C_{3}\right)_{*}$.

Theorem 9. Let $G$ and $H$ be almost completely decomposable groups such that $i(G)$ and $i(H)$ are powers of the same prime $p$, and neither $G$ nor $H$ has a rank 1 summand. Then $G \oplus H$ has no rank 1 summand. Any two 
decompositions of $G$ as a direct sum of indecomposable groups will involve the same number of rank 1 summands of each type $\tau$.

Proof. Suppose that $G \oplus H=A \oplus K$ where rank $A=1$ and $\mathfrak{t}(A)=\tau$. Let $C$ and $D$ be regulating subgroups of $G$ and $H$, and let $a$ be the projection onto $A$. Then $A \subseteq C_{\tau} \oplus D_{\tau} \oplus G^{\sharp}(\tau) \oplus H^{\sharp}(\tau)$. Let $C_{1}$ and $D_{1}$ be the components of $A$ in $C_{\tau}$ and $D_{\tau}$ and let $S=\{\sigma \mid \sigma \not \tau\}$. It follows from Lemma 8 that $a\left(\left(C_{1} \oplus G(S)\right)_{*}\right) / a\left(C_{1} \oplus G(S)_{*}\right)$ is trivial or a cyclic $p$-group. In the latter case, since $a\left(G(S)_{*}\right)=0, a\left(C_{1}\right) \subseteq p a(G)=p A \neq A$. Likewise either $\alpha\left(D_{1}\right)$ $\subseteq p A \neq A$ or $\alpha\left(D_{1}\right)=\alpha\left(\left(D_{1} \oplus H(S)\right)_{*}\right)$. But $\alpha\left(G^{\#}(\tau)\right)=\alpha\left(H^{\#}(\tau)\right)=0$, so $A=$ $a\left(C_{1}\right)+\alpha\left(D_{1}\right)$. Hence we may assume, say, that $\alpha\left(\left(C_{1} \oplus G(S)\right)_{*}\right)=a\left(C_{1}\right)$. This means that $\left(C_{1} \oplus G(S)\right)_{*} \subseteq C_{1} \oplus K$. Since $G(S)_{*} \subseteq K$, this means that $C_{1} \oplus$ $G(S)_{*}$ is pure, so, by Lemma $8, C_{1}$ is a summand of $G$, a contradiction. The last statement of the theorem now follows by applying the first part to the indecomposable summands of $G$ and using Corollary 7 .

3. K-equivalence. We will now characterize $K$-equivalence for almost completely decomposable groups. The most difficult part of the proof, given in the following lemma, applies to all finite rank groups.

Lemma 10. Let $G$ and $H$ be $K$-equivalent finite rank torsion free abelian groups. If $n$ is a positive integer, then $H$ contains a subgroup $G^{\prime}$ isomorphic to $G$ such that $\left[H: G^{\prime}\right]$ is prime to $n$.

Proof. We may suppose that $G$ and $H$ are reduced. For each positive integer $m$, there is a largest subgroup $G_{m}$ of $G$ so that $m ! G_{m}=G_{m}$. Each $G_{m}$ is pure, so for sufficiently large $m$, we have $G_{m}=G_{m+1}=\cdots$, hence $G_{m}$ is divisible, hence $G_{m}=0$. Without loss of generality, we may suppose that $n$ is a multiple of $m$ !. Hence $n A \neq A$ for every nonzero subgroup $A$ of $G$.

Now suppose $G \oplus L \approx H \oplus L$ where $L$ has finite rank. Let

$$
\mathrm{E}(G)=\operatorname{End}(G) / n \operatorname{End}(G)
$$

and for any group $A$ let

$$
\mathbf{H}(A)=\operatorname{Hom}(G, A) / n \operatorname{Hom}(G, A) .
$$

Then $\mathbf{H}(A)$ is a finite length right $\mathbf{E}(G)$-module and $\mathbf{H}$ is an additive functor. Hence $\mathbf{H}(G) \oplus \mathbf{H}(L) \approx \mathbf{H}(H) \oplus \mathbf{H}(L)$ and so $\mathbf{H}(G) \approx \mathbf{H}(H)$ by the Krull-Schmidt theorem . In particular, $\mathbf{H}(H)$ is cyclic. Let $\phi \in \operatorname{Hom}(G, H)$ represent a generator for $\mathbf{H}(H)$. Now for any group $A$, let $\mathrm{F}(A)$ be the subgroup of $A / n A$ generated by $(f(G)+n A) / n A$ for all $f \in \operatorname{Hom}(G, A)$. Then $\mathbf{F}$ is an additive functor, so that $\mathbf{F}(H) \approx \mathbf{F}(G)=G / n G$ by the Krull-Schmidt theorem, and we con- 
clude that $\mathbf{F}(H)=H / n H$, since $H / n H \approx G / n G$ and $H / n H$ is finite. Since clearly $\mathbf{F}(H)=(\phi(G)+n H) / n H$, we see that $\phi$ induces an isomorphism from $G / n G$ onto $H / n H$. From the fact that this induced map is monic we infer first that $\phi$ is monic, and second that $\phi(G) \cap n H=n \phi(G)$, so that $H / \phi(G)$ has no elements with order dividing $n$. Hence $G^{\prime}=\phi(G)$ is the desired subgroup.

Remarks. (1) It follows from Lemma 10 that if $G$ and $H$ are $K$-equivalent and $p G=G$ for all but finitely many primes $p$, then $G \approx H$.

(2) If $G$ and $H$ are $K$-equivalent, then by Lemma 10 there exist subgroups $G_{1} \approx G_{2} \approx G$ of $H$ so that $\left[H: G_{1}\right]$ and $\left[H: G_{2}\right]$ are relatively prime. It follows (as in the proof of Theorem 11) that $H$ is isomorphic to a summand of $G \oplus G$. In particular, if $G \oplus G$ has, up to isomorphism, only finitely many summands, then there are only finitely many such $H$.

Theorem 11. Let $G$ and $H$ be almost completely decomposable groups. Then the following statements are equivalent:

(i) $G$ and $H$ are $K$-equivalent.

(ii) $i(G)=i(H)$ and $H$ contains a subgroup $G^{\prime}$ with $G^{\prime} \approx G$ and $\left[H: G^{\prime}\right]$ finite and prime to $i(G)$.

(iii) $G \oplus L \approx H \oplus L$ where $L$ is isomorphic to a regulating subgroup of G.

Proof. (i) $\Rightarrow$ (ii). By Corollary 2 and Lemma 10 .

(ii) $\Rightarrow$ (iii). Let $n=i(G), m=\left[H: G^{\prime}\right]$ and choose integers $r$ and $s$ so that $r m+s n=1$. Let $D$ be a regulating subgroup of $H$ and map $H$ into $G^{\prime} \oplus$ $D$ by $h \mapsto(r m h, s n h)$ and map $G^{\prime} \oplus D$ onto $H$ by $(x, y) \mapsto x+y$. Since $r m b+$ $s n h=h, H$ has been embedded as a summand in $G^{\prime} \oplus D$. If $L$ is a complementary summand, then $L$ and $D$ are quasi-isomorphic, so $L$ is almost completely decomposable. By Corollary $3, i(L)=i(D)=1$, so $L$ is completely decomposable. Thus $L \approx D$.

(iii) $\Rightarrow$ (i). Obvious.

Remark. It can in fact be shown that if $G$ and $H$ are $K$-equivalent almost completely decomposable groups of rank $r$, and if $L$ is isomorphic to a rank $r-1$ summand of a regulating subgroup of $G$, then $G \oplus L \approx H \oplus L$. The proof is tedious and computational and will not be given here.

\section{REFERENCES}

1. D. M. Arnold and E. L. Lady, Endomorphism rings and direct sums of torsion free abelian groups, Trans. Amer. Math. Soc. (to appear).

2. L. Fuchs, Infinite abelian groups. Vol. I, Pure and Appl. Math., vol. 36, Academic Press, New York, 1970 and 1973. MR 41 \#333. 